\title{
Peak Energy Correlations for Gamma-Ray Bursts
}

\author{
Walid J. Azzam \\ Department of Physics, College of Science, University of Bahrain, Sakhir, Bahrain \\ Email:wjazzam@uob.edu.bh,wjazzam@gmail.com
}

How to cite this paper: Azzam, W.J. (2017) Peak Energy Correlations for Gamma-Ray Bursts. Journal of Applied Mathematics and Physics, 5, 1515-1520. https://doi.org/10.4236/jamp.2017.58124

Received: July 13, 2017

Accepted: July 31, 2017

Published: August 4, 2017

Copyright $\odot 2017$ by author and Scientific Research Publishing Inc. This work is licensed under the Creative Commons Attribution International License (CC BY 4.0).

http://creativecommons.org/licenses/by/4.0/

\begin{abstract}
Gamma-ray bursts (GRBs) are the most powerful explosions in the universe. Over the past two decades, several GRB energy and luminosity correlations were discovered. These correlations typically involve an observable parameter, like the observed peak energy, $E_{p, o b s}$, and a non-observable quantity, like the equivalent isotropic energy, $E_{i s o}$. This paper provides a brief review of GRB peak energy correlations. Specifically, it focuses on the Amati relation, which correlates $E_{p, o b s}$ and $E_{i s o}$ and the Ghirlanda relation, which correlates $E_{p, o b s}$ and $E_{p}$ the total energy corrected for beaming. The paper also discusses the physical interpretation of these relations in the context of the internal shock model.
\end{abstract}

\section{Keywords}

Gamma-Ray Bursts, Peak Energy Correlations, Energy Indicators

\section{Introduction}

Gamma-ray bursts (GRBs) are extremely powerful stellar explosions with an equivalent isotropic energy, $E_{i s o}$, that can exceed $10^{54} \mathrm{erg}$ [1]. Their light curves consist of intense irregular pulses that typically last for a few seconds and their spectra are nonthermal peaking between 10 and $10^{4} \mathrm{keV}$. The radiation produced by GRBs is believed to emanate from jets, but the precise mechanism behind the formation of these jets is still not fully understood [2].

Over the past two decades, several GRB energy and luminosity correlations were discovered. Some were obtained from the light curves, like the time-lag and variability relations [3] [4], while others were obtained from the spectra and include the Amati relation [5] [6] [7] [8], the Ghirlanda relation [9], the Yonetoku relation [10] [11], and the Liang-Zhang relation [12]. These correlations are important because they can potentially be used as cosmological probes to constrain 
cosmological parameters [12]-[18], and also as tools that might shed light on the physics of GRBs [19] [20].

This paper provides a review of the GRB energy correlations that involve the peak energy, $E_{p, o b s}$, which is the peak energy observed in the $v F_{V}$ spectrum. Section 2 provides a brief background on how the correlations involving $E_{p, o b s}$ were first noticed, and Sections 3 and 4 focus, respectively, on two important correlations: the Amati relation and the Ghirlanda relation. This is followed by a discussion of the importance and physical interpretation of these correlations in Section 5, and our conclusions are provided in Section 6.

\section{Peak Energy Correlations}

GRB correlations involving the peak energy were first noticed in 1995 by [21], who studied 399 GRBs observed by the BATSE instrument and discovered a correlation between $E_{p, o b s}$ and the peak flux, $F_{p}$. They calculated $F_{p}$ from the photon count data in the $50-300 \mathrm{keV}$ energy band and the $256 \mathrm{~ms}$ time bin. They then selected those bursts with $F_{p}>1$ photon $\cdot \mathrm{cm}^{-2} \cdot \mathrm{s}^{-1}$ and divided them into 5 bins of varying width, each with about 80 bursts. They found a correlation between the mean observed peak energy, $\left\langle E_{p, o b s}\right\rangle$, and the logarithm of $F_{p}$ with a statistical significance of $\rho=0.90$ and $P=0.04$.

In 2000, a study by [22] found a strong correlation between $E_{p, o b s}$ and the bolometric fluence, $S_{\text {too }}$ in the same energy range as [21]. They expressed the correlation as:

$$
\log \left(E_{p, o b s}\right) \approx 0.29 \log \left(S_{\text {tot }}\right),
$$

with a Kendall correlation coefficient $\tau=0.80$ and a chance probability $P=10^{-13}$. However, it is important to keep in mind that their selection criteria, $F_{p}>3$ photons $\cdot \mathrm{cm}^{-2} \cdot \mathrm{s}^{-1}$ and $S_{\text {tot }}>5 \times 10^{-6} \mathrm{erg} \cdot \mathrm{cm}^{-2}$, included only the most luminous bursts. The correlation discovered by [22] was the basis for later studies that led to the discovery of two important correlations: the Amati relation and the Ghirlanda relation.

\section{The Amati Relation}

The peak energy correlations found by [21] and [22] were in the observer frame due to the paucity of data points with known redshift. The first rest-frame correlation involving the intrinsic peak energy, $E_{p, i}$ was found by [5] in 2002 and is referred to as the Amati relation. The study by [5] was based on 12 bursts, detected by BeppoSAX, with known redshifts, $z$. The intrinsic peak energy is calculated from the observed one using:

$$
E_{p, i}=(1+z) \cdot E_{p, o b s} .
$$

On the other hand, $E_{i s o}$ can be calculated from the bolometric flux using:

$$
E_{\text {iso }}=4 \pi d^{2} S_{\text {tot }} /(1+z) \text {, }
$$

where $d$ is the luminosity distance, which can be calculated from $z$ after assum- 
ing a certain cosmological model. In Amati's original paper [5], a flat universe was assumed with $\Omega_{M}=0.3, \Omega_{\Lambda}=0.7$, and $H_{0}=65 \mathrm{~km} \cdot \mathrm{s}^{-1} \cdot \mathrm{Mpc}^{-1}$. The Amati relation can be expressed logarithmically as:

$$
\log \left(E_{\text {iso }}\right)=A+B \cdot \log \left(E_{p, i} /\left\langle E_{p, i}\right\rangle\right),
$$

where the normalization, $A$, and the slope, $B$, are constants, and where $\left\langle E_{p, i}\right\rangle$ is the mean value of the intrinsic peak energy for the entire data sample. The approximate mean values for the fitting parameters are $\langle A\rangle \approx 53$ and $\langle B\rangle \approx 1$. Alternatively, the Amati relation can be expressed as:

$$
E_{p, i}=K \cdot\left(E_{i s o} / 10^{52} \mathrm{erg}\right)^{m}
$$

where $E_{p, i}$ is in $\mathrm{keV}$, and $K$ and $m$ are constants. In Amati's original study [5], $m \approx$ 0.5 and $K \approx 95$. However, more recent studies [23] [24] [25] [26] [27] found mean values of $\langle m\rangle=0.45$ and $\langle K\rangle=141$.

\section{The Ghirlanda Relation}

The Ghirlanda relation is a correlation between the peak energy and the total energy corrected for beaming, $E_{p}$ which is given by:

$$
E_{\gamma}=\left[1-\cos \left(\theta_{j e t}\right)\right] \cdot E_{i s o},
$$

where $\theta_{j e t}$ is the jet's half-opening angle. This correlation was discovered in 2004 by [9] who used 40 GRBs with known $E_{i s o}$ and $z$. According to [28], $\theta_{j e t}$ can be calculated (in degrees) as follows:

$$
\theta_{\text {jet }}=0.161\left[T_{\text {break }} /(1+z)\right]^{3 / 8}\left[n \cdot n_{\gamma} \cdot E_{\text {iso }}\right]^{1 / 8},
$$

where $T_{\text {break }}$ (measured in days) is the time for the power-law break in the afterglow light curve, $n_{\gamma}$ is the radiative efficiency, $n$ is the density of the circumburst medium (in particles $/ \mathrm{cm}^{3}$ ), and $E_{i s o}$ is measured in units of $10^{52} \mathrm{erg}$. To compute $T_{\text {break }}$ properly, several issues should be kept in mind [29]:

- The jet break should be detected in the optical window

- The optical light curve should not end at $T_{\text {break }}$ but should continue beyond it

- The flux from the host galaxy and from any probable supernova should be subtracted out

After considering the above points, the Ghirlanda relation can be expressed as [29]:

$$
\log \left(E_{\text {peak }} / 100 \mathrm{keV}\right)=(0.48 \pm 0.02)+(0.70 \pm 0.04) \times \log \left[E_{\gamma} / 4.4 \times 10^{50} \mathrm{erg}\right] .
$$

\section{Physical Interpretation}

The first attempt to provide a physical interpretation of correlations involving $E_{\text {peak }}$ was carried out by [22] who investigated the $E_{\text {peak }}-S_{\text {tot }}$ correlation. According to their study, this correlation can be obtained rather easily by assuming a thin synchrotron radiation process by a power law distribution of electrons with a Lorentz factor, $\Gamma$, that exceeds some minimum value, $\Gamma_{M}$. Moreover, they 
found that the internal shock model gave a tighter $E_{\text {peak }}-S_{\text {tot }}$ correlation than the external shock model.

The above results were confirmed by [5] who showed that the $E_{\text {peak }}-E_{\text {iso }}$ correlation (the Amati relation) can be obtained by assuming an optically thin synchrotron shock model with an electron distribution given by: $N(\Gamma)=N_{0}(\Gamma)^{-\beta}$, for $\Gamma>\Gamma_{M}$, where $\beta$ is the power law index. However, [5] assumed that $N_{0}$ and the burst duration are constants, which is not completely justified because GRBs clearly have varying durations.

A recent study [30] investigated whether the $E_{\text {peak }}-E_{\text {iso }}$ correlation can be obtained in the context of the internal shock model but through the impact of only two shells. The study involved both simulated $E_{\text {peak }}-E_{\text {iso }}$ distributions and observed data (for 58 bursts), and it included only bright $S$ wift GRBs with $F_{p}>2.6$ photons $\cdot \mathrm{cm}^{-2} \cdot \mathrm{s}^{-1}$ in the $15-150 \mathrm{keV}$ energy band. The results indicated that the $E_{\text {peak }}-E_{\text {iso }}$ correlation can be obtained theoretically but under certain restrictions. First, most of the dispersed energy should be radiated via a few electrons. Second, the range in the Lorentz factors used should be tight. Finally, the variability timescale for $\Gamma$ should scale with the mean value of $\Gamma$. Concerning the Ghirlanda relation, the theoretical study by [31] showed that this relation can be obtained theoretically if one assumes that $\Gamma$ and $\theta_{j e t}$ are inversely proportional. More specifically, they found that:

$$
\left[\theta_{j e t, \max }\right]^{2.5} \cdot \Gamma_{\max }=\text { constant. }
$$

\section{Conclusion}

The peak energy correlations of GRBs are important relations that can be utilized to probe the physics of GRBs. The most important peak energy correlations are the Amati relation, which correlates the peak energy and $E_{i s o}$, and the Ghirlanda relation, which correlates the peak energy and $E_{\gamma}$. Both relations can be understood theoretically in the context of the internal shock model, but there are important assumptions that should be kept in mind. When calibrated properly, these relations can be employed as tools to probe different cosmological models and also to probe the underlying physics behind GRBs.

\section{Acknowledgements}

The author would like to thank the anonymous referee for the feedback that helped improve the paper.

\section{References}

[1] Atteia, J.-L., et al. (2017) The Maximum Isotropic Energy of Gamma-Ray Bursts. The Astrophysical Journal, 837, 119. https://doi.org/10.3847/1538-4357/aa5ffa

[2] Le, T. and Mehta, V. (2017) Revisiting the Redshift Distribution of Gamma-Ray Bursts in the Swift Era. The Astrophysical Journal, 837, 17. https://doi.org/10.3847/1538-4357/aa5fa7

[3] Norris, J.P., et al. (2000) Connection between Energy Dependent Lags and Peak 
Luminosity in Gamma-Ray Bursts. The Astrophysical Journal, 534, 248-257. https://doi.org/10.1086/308725

[4] Fenimore, E.E. and Ramirez-Ruiz, E. (2000) Redshifts for 220 BATSE Gamma-Ray Bursts Determined by Variability and the Cosmological Consequences. e-print astro-ph/0004176.

[5] Amati, L., et al. (2002) Intrinsic Spectra and Energetics of BeppoSAX Gamma-Ray Bursts with Known Redshifts. Astronomy and Astrophysics, 390, 81-89. https://doi.org/10.1051/0004-6361:20020722

[6] Amati, L. (2006) The $E_{p, i}-E_{i s o}$ Correlation in Gamma-Ray Bursts: Updated Observational Status, Re-Analysis and Main Implications. Monthly Notices of the Royal Astronomical Society, 372, 233-245.

https://doi.org/10.1111/j.1365-2966.2006.10840.x

[7] Amati, L., et al. (2008) Measuring the Cosmological Parameters with the $E_{p, i}-E_{i s o}$ Correlation of Gamma-Ray Bursts. Monthly Notices of the Royal Astronomical Society, 391, 577-584. https://doi.org/10.1111/j.1365-2966.2008.13943.x

[8] Amati, L., et al. (2009) Extremely Energetic Fermi Gamma-Ray Bursts Obey Spectral Energy Correlations. Astronomy and Astrophysics, 508, 173-180.

https://doi.org/10.1051/0004-6361/200912788

[9] Ghirlanda, G., et al. (2004) The Collimation-Corrected Gamma-Ray Burst Energies Correlate with the Peak Energy of their $v F_{v}$ Spectrum. The Astrophysical Journal, 616, 331-338. https://doi.org/10.1086/424913

[10] Yonetoku, D., et al. (2004) Gamma-Ray Burst Formation Rate Inferred from the Spectral Peak Energy - Peak Luminosity Relation. The Astrophysical Journal, 609, 935-951. https://doi.org/10.1086/421285

[11] Ghirlanda, G., et al. (2010) Spectral-Luminosity Relation within Individual Fermi Gamma-Ray Bursts. Astronomy and Astrophysics, 511, A43-A53. https://doi.org/10.1051/0004-6361/200913134

[12] Liang, E. and Zhang, B. (2005) Model-Independent Multivariable Gamma-Ray Burst Luminosity Indicator and Its Possible Cosmological Implications. The Astrophysical Journal, 633, L611-L623. https://doi.org/10.1086/491594

[13] Ghirlanda, G., et al. (2006) Cosmological Constraints with GRBs: Homogeneous vs. Wind Density Profile. Astronomy and Astrophysics, 452, 839-844. https://doi.org/10.1051/0004-6361:20054544

[14] Capozziello, S. and Izzo, L. (2008) Cosmography by Gamma-Ray Bursts. Astronomy and Astrophysics, 490, 31-36. https://doi.org/10.1051/0004-6361:200810337

[15] Demianski, M. and Piedipalumbo, E. (2011) Standardizing the GRBs with the Amati $E_{p, i}-E_{i s o}$ Relation: the Updated Hubble Diagram and Implications for Cosmography. Monthly Notices of the Royal Astronomical Society, 415, 3580-3590. https://doi.org/10.1111/j.1365-2966.2011.18975.x

[16] Azzam, W.J. and Alothman, M.J. (2006) Constraining Cosmological Parameters through Gamma-Ray Bursts. Advances in Space Research, 38, 1303-1306. https://doi.org/10.1016/j.asr.2004.12.019

[17] Azzam, W.J. and Alothman, M.J. (2006) Gamma-Ray Burst Spectral-Energy Correlations as Cosmological Probes. IL Nuovo Cimento B, 121, 1431-1432. https://doi.org/10.1393/ncb/i2007-10270-5

[18] Dai, Z.G., Liang, E.W. and Xu, D. (2004) Constraining $\Omega_{\mathrm{M}}$ and Dark Energy with Gamma-Ray Bursts. The Astrophysical Journal, 612, L101-L104.

https://doi.org/10.1086/424694 
[19] Zhang, B. and Mészáros, P. (2002) An Analysis of Gamma-Ray Burst Spectral Break Models. The Astrophysical Journal, 581, 1236-1247. https://doi.org/10.1086/344338

[20] Thompson, C., Mészáros, P. and Rees, M.J. (2007) Thermalization in Relativistic Outflows and the Correlation between Spectral Hardness and Apparent Luminosity in Gamma-Ray Bursts. The Astrophysical Journal, 666, 1012-1023. https://doi.org/10.1086/518551

[21] Mallozi, R.S., et al. (1995) Gamma-Ray Bursts Spectra and the Hardness-Intensity Correlation. Proceedings of Gamma-Ray Bursts 4th Huntsville Symposium, AIP Conference Series, 428, 273-277. https://doi.org/10.1063/1.55334

[22] Lloyd, N.M., Petrosian, V. and Mallozi, R.S. (2000) Cosmological versus Intrinsic: The Correlation between Intensity and the Peak of the $v F_{v}$ Spectrum of GammaRay Bursts. The Astrophysical Journal, 534, 227-238. https://doi.org/10.1086/308742

[23] Azzam, W.J. and Alothman, M.J. (2013) Redshift Independence of the Amati and Yonetoku Relations for Gamma-Ray Bursts. International Journal of Astronomy and Astrophysics, 3, 372-375. http://dx.doi.org/10.4236/ijaa.2013.34042

[24] Zitouni, H., Guessoum, N. and Azzam, W.J. (2014) Revisiting the Amati and Yonetoku Relations with Swift GRBs. Astrophysics and Space Science, 351, 267-279. https://doi.org/10.1007/s10509-014-1839-5

[25] Azzam, W.J. (2016) A Brief Review of the Amati Relation for GRBs. International Journal of Astronomy and Astrophysics, 6, 378-383. http://dx.doi.org/10.4236/ijaa.2016.64030

[26] Zitouni, H., Guessoum, N. and Azzam, W.J. (2016) Determination of Cosmological Parameters from Gamma-Ray Burst Characteristics and Afterglow Correlations. Astrophysics and Space Science, 361, 383. https://doi.org/10.1007/s10509-016-2969-8

[27] Azzam, W.J. and Al Dallal, S. (2015) Gamma-Ray Bursts: Origin, Types, and Prospects. Journal of Magnetohydrodynamics and Plasma Research, 20, 367.

[28] Sari, R., Piran, T. and Halpern, J.P. (1999) Jets in Gamma-Ray Bursts. The Astrophysical Journal, 519, L17-L20. https://doi.org/10.1086/312109

[29] Dainotti, M.G., Del Vecchio, R. and Tarnopolski, M. (2016) Gamma-Ray Burst Prompt Correlations.

[30] Mochkovitch, R. and Nava, L. (2015) The $E_{p}-E_{i s o}$ Relation and the Internal Shock Model. Astronomy and Astrophysics, 577, A31. https://doi.org/10.1051/0004-6361/201424490

[31] Ghirlanda, G., et al. (2013) The Faster the Narrower: Characteristic Bulk Velocities and Jet Opening Angles of Gamma-Ray Bursts. Monthly Notices of the Royal Astronomical Society, 428, 1410-1423. https://doi.org/10.1093/mnras/sts128 
Submit or recommend next manuscript to SCIRP and we will provide best service for you:

Accepting pre-submission inquiries through Email, Facebook, LinkedIn, Twitter, etc. A wide selection of journals (inclusive of 9 subjects, more than 200 journals)

Providing 24-hour high-quality service

User-friendly online submission system

Fair and swift peer-review system

Efficient typesetting and proofreading procedure

Display of the result of downloads and visits, as well as the number of cited articles Maximum dissemination of your research work

Submit your manuscript at: http://papersubmission.scirp.org/

Or contact jamp@scirp.org 